\title{
A Mouse Model of Sural Nerve Injury-Induced Neuropathy: Gabapentin Inhibits Pain-Related Behaviors and the Hyperactivity of Wide-Dynamic Range Neurons in the Dorsal Horn
}

\author{
Yu Omori ${ }^{1}$, Kenta Kagaya ${ }^{1}$, Ryugo Enomoto ${ }^{1}$, Atsushi Sasaki ${ }^{1}$, Tsugunobu Andoh ${ }^{1}$, Hiroshi Nojima ${ }^{2}$, \\ Hiroki Takahata ${ }^{3}$, and Yasushi Kuraishi ${ }^{1, *}$ \\ ${ }^{I}$ Department of Applied Pharmacology, Graduate School of Medicine and Pharmaceutical Sciences, University of Toyama, \\ 2630 Sugitani, Toyama 930-0194, Japan \\ ${ }^{2}$ Department of Pharmacology, School of Pharmaceutical Sciences, Ohu University, \\ 31-1 Misumido, Koriyama, Fukushima 963-8611, Japan \\ ${ }^{3}$ Department of Pharmaceutical Chemistry, Tohoku Pharmaceutical University, Sendai 981-8558, Japan
}

Received November 29, 2008; Accepted January 28, 2009

\begin{abstract}
This study was conducted to make a new mouse model of neuropathic pain due to injury to a branch of the sciatic nerve. One of three branches (sural, tibial, and common peroneal nerves) of the sciatic nerve was tightly ligated, and mechanical and cool stimuli were applied to the medial part (tibial and common peroneal nerve territories) of the plantar skin. The three types of nerve injuries produced behavioral mechanical hypersensitivities, and the extent of the hypersensitivities after sural and tibial nerve ligation was larger than that of common peroneal nerve ligation. Sural nerve ligation did not affect motor function of the affected hind paw, but tibial and common peroneal nerve ligation produced motor dysfunction. These results suggest that the ligation of the sural nerve is the most suitable for behavioral study. Sural nerve ligation induced behavioral hypersensitivities to mechanical and cool stimuli, which were almost completely inhibited by gabapentin $(30 \mathrm{mg} / \mathrm{kg})$. Sural nerve ligation increased spontaneous activity and responses of the wide-dynamic range neurons in the lumbar dorsal horn, which were also almost completely inhibited by gabapentin $(30 \mathrm{mg} / \mathrm{kg})$. Sural nerve ligation provides a new mouse model of neuropathic pain, which is easy to prepare and sensitive to gabapentin.
\end{abstract}

Keywords: neuropathic pain, gabapentin, sural nerve-ligation injury, cold allodynia, mechanical allodynia

\section{Introduction}

Advances in the understanding of clinical pain have been facilitated by the development of animal models that reflect some component of clinical pain syndromes. Neuropathic pain is generally difficult to treat and several animal models of nerve injury-induced pain have been developed for the study of neuropathic pain. Animal models generally used are made by the constriction or partial ligation of the sciatic nerve or the tight ligation of the spinal nerve $(1-3)$. These three models

*Corresponding author. kuraisiy@pha.u-toyama.ac.jp Published online in J-STAGE on April 4, 2009 (in advance) doi: 10.1254/jphs.08319FP have distinct pain features that are different from each other $(4,5)$, suggesting that mechanisms of neuropathic pain differ depending on the site and mode of nerve injury. In humans, persistent pain may be caused by trauma to distal nerve branches as well as nerve trunks, such as injury to distal nerve branches of the lower leg $(6,7)$. Therefore, animal models of neuropathic pain produced by injury to the distal branch of the sciatic nerve are needed for investigating the mechanisms of neuropathic pain of distal nerve branches. A few studies have developed rodent models of neuropathic pain with injuries to branches (tibial, common peroneal, and sural) of the sciatic nerve. The tight ligation of the tibial and common peroneal nerves with the sural nerve intact induces the increase of mechanical sensitivity and 
thermal responsiveness, especially in the sural territory, in rats (8). The tibial and common peroneal territories (central and medial regions of the plantar side, respectively) are suitable for mechanical and thermal stimulation in mice. The ligation of the tibial and common peroneal nerves induced mechanical allodynia in the sural territory in mice also, but allodynia was not observed in the tibial territory (9). The ligation of the sural and common peroneal nerves or the tibial nerve only does not induce allodynia in the tibial territory (9). In our preliminary experiments, the ligation of the tibial and common peroneal nerves exerted obvious effects on the posture and motor functions of the hind paw, which might affect the evaluation of pain-like responses of the paw. In this study, therefore, we compared the effects of the tight ligation of one of three branches of the sciatic nerve on pain-related responses to the stimulation of the tibial and common peroneal territories and the motor function and appearance of the hind paw. We show here that the ligation injury of the sural nerve is the most suitable for behavioral study.

Gabapentin, an anticonvulsant, has been shown to be effective against neuropathic pain $(10,11)$. It inhibits brush-induced and cold allodynia in patients with neuropathic pain (12). In animal experiments, gabapentin suppresses mechanical and cold allodynia in rats with spinal nerve ligation (13). On the other hand, it inhibits mechanical allodynia without effects on cold allodynia and mechanical hyperalgesia in rats which undergo the ligation of the tibial and common peroneal nerves (14), and it does not affect mechanical allodynia in rats with ligation of the tibial nerve (15). These findings suggest that the efficacy of gabapentin is dependent on the type of surgical operation and the branches of sciatic nerve ligated. Therefore, in this study, we examined the effects of gabapentin on pain-related behaviors and the hyperactivity of the dorsal horn neurons to mechanical and cool stimuli in mice that underwent ligation injury of the sural nerve.

\section{Materials and Methods}

\section{Animals}

Male ICR mice (Japan SLC, Shizuoka), five-weeksold, at the start of experiments were used. They were housed 4-6 per cage in a room under controlled temperature $\left(22 \pm 1^{\circ} \mathrm{C}\right)$, humidity $(55 \pm 10 \%)$, and a 12 h light / 12-h dark cycle (lights on 07:00-19:00 h). Food and water were available ad libitum. This study was approved by the Committee for Animal Experiments at University of Toyama. The experiments were done in accordance with animal experiment guidelines of The Japanese Pharmacological Society. The pain test was performed according to the guidelines on ethical standards for investigations of experimental pain in animals (16).

\section{Surgical operation}

The animals were randomly assigned to naive, shamoperated, sural nerve-injured, tibial nerve-injured, or common peroneal nerve-injured groups. Under ether anesthesia, the skin of the lateral thigh was incised and the sciatic nerve and its three branches were exposed through the biceps femoris muscle (8). The sural, tibial, or common peroneal nerve was ligated with a 9-0 silk suture and the wound was closed with a 7-0 silk suture. The sham-operated group was given all procedures except ligation. When extension of the hind leg, flexion of the toes, and/or dragging of the hind leg were observed, motor function was considered abnormal.

\section{Pain test}

Tactile allodynia and mechanical hyperalgesia of the hind paw were assessed as described (17). The mice were placed individually in a plastic cage $(8 \times 10 \times$ $18 \mathrm{~cm}$ ) with a wire mesh bottom. After at least 15 -min acclimation period, von Frey filaments with bending force of $1.6 \mathrm{mN}$ (innocuous stimulation) or $9.8 \mathrm{mN}$ (noxious stimulation) were pressed perpendicularly against the medial part (tibial and common peroneal nerve territories) of the plantar skin and held for $2 \mathrm{~s}$ with it slightly buckled. The responses to these stimuli were ranked as follows: 0 , no response; 1 , move away from the filament; 2, immediate flinching or licking of the hind paw. The stimulation of the same intensity was applied six times to each hind paw at intervals of several seconds and an average of six values served as the painrelated score.

Cool stimulation was performed by applying acetone $(5 \mu \mathrm{l})$ to the medial part of the plantar skin. In our preliminary experiments, application of acetone evoked a quick and transient withdrawal response even in naive mice and sustained flinching and licking behaviors in sural nerve-ligated mice; therefore, flinching and licking behaviors were considered pain-related behaviors. The test was done five times at about 1-min intervals, and the number of positive tests served as the painrelated score. In the presented results, the effects of gabapentin pain-related score was normalized to the score of the affected paw before gabapentin injection.

\section{Electrophysiological recording}

The mice were anesthetized with sodium pentobarbital ( $80 \mathrm{mg} / \mathrm{kg}$, i.p. initially, with additional as necessary). A laminectomy was carried out to expose the spinal cord at the levels of T13 to L2 vertebrae, and the animal was 
mounted in a stereotaxic frame. The cord was held rigidly with clamps directly rostral and caudal to this area. A recording tungsten electrode $(10-12 \mathrm{M} \Omega$ resistance) advanced with a pulse motor microdrive was inserted into the dorsal horn $300-500 \mu \mathrm{m}$ below the dorsal surface, and units responsive to innocuous mechanical stimulation of the ipsilateral hind paw were searched. When isolated, units were classified as widedynamic range (WDR) neurons if they responded in an increased fashion to brushing, pressing, and pinch of the receptive field for $2 \mathrm{~s}$. The response of the WDR neuron was evaluated by the frequency of evoked firing during mechanical stimulation or $0.5-10 \mathrm{~s}$ (the period corresponding to delayed behavior) after acetone application. The spontaneous activity of neurons was recorded for $10 \mathrm{~min}$ before application of these stimuli. These experiments were done 7-14 days after sural nerve ligation because pain-related behaviors were marked and relatively constant during this period.

\section{Agent}

Gabapentin, synthesized by H. Takahata, was dissolved in saline and $\mathrm{pH}$ was adjusted to $7.0-7.4$ with $1 \mathrm{M} \mathrm{NaOH}$. It was administered intraperitoneally in a volume of $0.1 \mathrm{ml} / 10 \mathrm{~g}$ body weight.

\section{Data analyses}

Data are each expressed as the mean \pm S.E.M. Neuronal activity was collected and analyzed using a software Spike Histogram (PowerLab/8s; AD Instruments Pty, Castle Hill, Australia). Data on the time course of analgesic effects were analyzed with Friedman repeated measures analysis of variance on ranks followed by Dunnett's test. Other data were analyzed with Student's $t$-test or Wilcoxon signed rank test. $P<0.05$ was considered to be significant.

\section{Results}

Behavioral effects of ligation of the branches of the sciatic nerve

Sham operation did not affect the response to the mechanical stimulation with von Frey filaments (Fig. 1A). Ligation of the sural nerve resulted in rapid increase in responses to mechanical stimuli; the responses started to increase at $2 \mathrm{~h}$, peaked at $2-9$ days, and subsided 50 days after ligation (Fig. 1B). The ligation of the tibial nerve gradually increased responses to mechanical stimuli; the responses gradually increased until 11-13 days after ligation and then gradually returned to normal by $50-60$ days (Fig. 1C). The ligation of the common peroneal nerve produced an increase in responses to mechanical stimuli after a transient

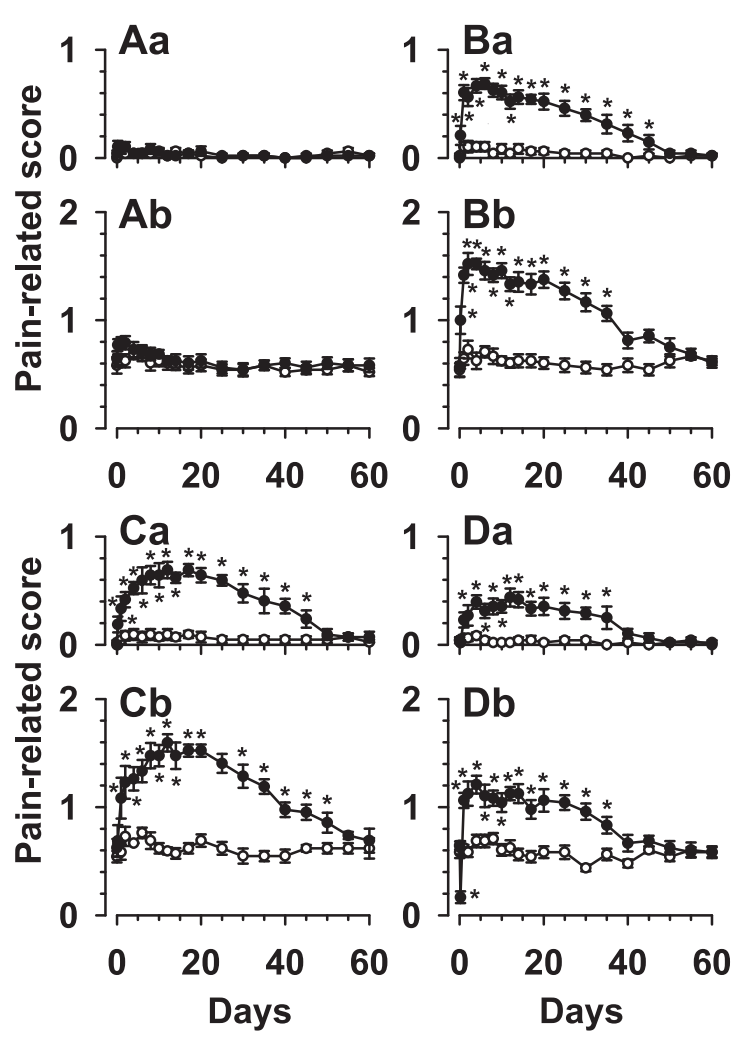

Fig. 1. Effects of the ligation of the branches of sciatic nerve on pain-related behavioral responses to mechanical stimulation with von Frey filaments. The mouse was given sham operation (A) or the tight ligation of sural (B), tibial (C), or common peroneal nerve (D). The response of the hind paw on the treated (closed circles) and contralateral (open circles) sides to stimulation with von Frey filaments of bending force of 1.6 (a) or $9.8 \mathrm{mN}$ (b) were plotted against the day after surgical operation; the details of scoring procedures are described in Materials and Methods. Each value is the mean \pm S.E.M. of $7-8$ animals. ${ }^{*} P<0.05$ vs. day 0 (Dunnett's multiple comparisons).

unresponsiveness; the responses were not observed $2 \mathrm{~h}$ after ligation, increased from $24 \mathrm{~h}$ to 7 days, and subsided by 40 days (Fig. 1D). The peak responses were similar between mice with tibial nerve and sural nerve ligations, but those after common peroneal nerve ligation were smaller than those of the other ligations (Fig. 1: B - D).

The ligation of either tibial nerve or common peroneal nerve caused apparent abnormalities in motor function such as extension of the hind leg, flexion of the toes, and dragging of the hind leg. Figure 2 shows the hind paw on the treated side one day after the ligation of sciatic nerve branches. The form of the hind leg and toes was similar between sural nerve-ligated and sham-operated mice (Fig. 2: A and B). They walked normally (Table 1). In mice given the tibial nerve ligation, the hind leg extended and toes bent toward the plantar (Fig. 2C). They dragged the treated hind leg. These abnormalities were marked $2 \mathrm{~h}$ and one day after the operation and 


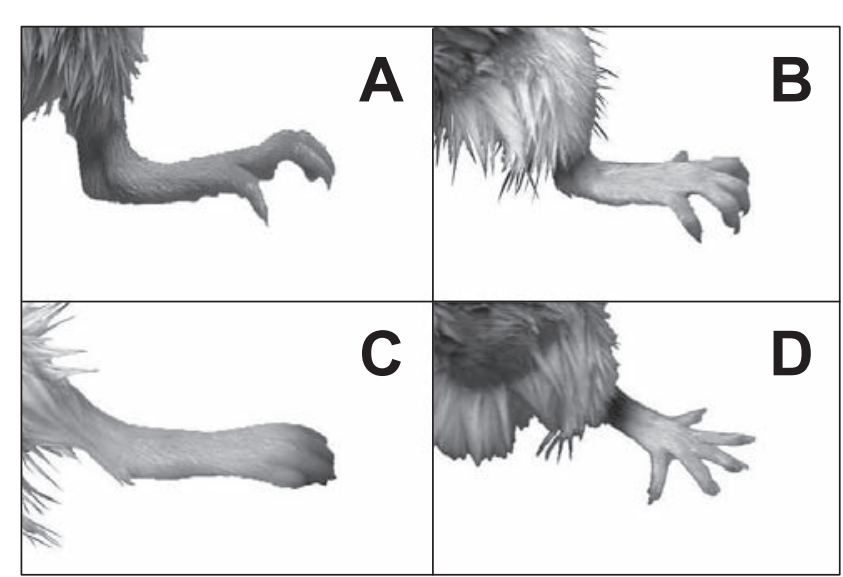

Fig. 2. Effects of the ligation of the branches of sciatic nerve on the form of the hind paw. The photos show typical examples one day after sham operation (A) and the tight ligation of sural (B), tibial (C), or common peroneal nerve (D).

gradually decreased until they were cured by 7 days (Table 1). In mice given the ligation of the common peroneal nerve, the toes appeared normal but the hind leg markedly extended (Fig. 2D). They dragged the treated hind leg. These abnormalities were marked from $2 \mathrm{~h}$ to 5 days after the operation and gradually decreased until they were cured by 20 days (Table 1). There were no clear differences in food intake and a gain of weight between the four groups (data not shown). Thus, ligation of the sural nerve produced marked pain-related responses to mechanical stimulation without marked motor abnormalities. Table 2 shows the effects of sural nerve ligation on responses to mechanical stimuli assessed by three different experimenters. Although response scores are different between the experimenters, the significant effects of the ligation were observed by all the experimenters. Thus, we used mice given the sural nerve ligation in the following experiments.

\section{Behavioral effects of gabapentin}

Since pain-related responses to mechanical stimuli peaked $2-9$ days after sural nerve ligation, the effects of gabapentin on pain-related responses were tested on day 7 after ligation. The ligation caused pain-related response to cool as well as mechanical stimuli (Fig. 3). Gabapentin (30, but not 3 and 10, $\mathrm{mg} / \mathrm{kg}$ ) inhibited these responses, which peaked at $2 \mathrm{~h}$ and almost subsided by $4 \mathrm{~h}$ (Fig. 3). Gabapentin $(3-30 \mathrm{mg} / \mathrm{kg})$ did not affect the responses of the contralateral hind paw (the effects of $30-\mathrm{mg} / \mathrm{kg}$ dose are shown in Fig. 3).

\section{Effects of sural nerve ligation on the activity of dorsal horn neurons}

The mean level of spontaneous activity of the WDR neurons in sural nerve-ligated mice was $2.2 \pm 0.4 \mathrm{~Hz}$ $(\mathrm{n}=12)$, which was significantly higher $(P<0.05)$ than that observed in naive ones $(0.6 \pm 0.1 \mathrm{~Hz}, \mathrm{n}=8)$. The

Table 1. Abnormality of the hind paw on the treated side

\begin{tabular}{lcccccccc}
\hline Time after operation & $2 \mathrm{~h}$ & $1 \mathrm{~d}$ & $3 \mathrm{~d}$ & $5 \mathrm{~d}$ & $7 \mathrm{~d}$ & $10 \mathrm{~d}$ & $14 \mathrm{~d}$ & $20 \mathrm{~d}$ \\
\hline Ligated nerve & & \multicolumn{7}{c}{ Number of animals (abnormal/observed) } \\
\hline Sham & $1 / 8$ & $0 / 8$ & $0 / 8$ & $0 / 8$ & $0 / 8$ & $0 / 8$ & $0 / 8$ & $0 / 8$ \\
Sural & $1 / 8$ & $0 / 8$ & $0 / 8$ & $0 / 8$ & $0 / 8$ & $0 / 8$ & $0 / 8$ & $0 / 8$ \\
Tibial & $7 / 7$ & $7 / 7$ & $6 / 7$ & $3 / 7$ & $0 / 7$ & $0 / 7$ & $0 / 7$ & $0 / 7$ \\
Common peroneal & $8 / 8$ & $8 / 8$ & $8 / 8$ & $8 / 8$ & $5 / 8$ & $5 / 8$ & $2 / 8$ & $0 / 8$ \\
\hline
\end{tabular}

When the mouse showed extension of the hind leg, flexion of the toe, and/or dragging the hind leg, its motor function was considered abnormal. The number of mice in the tibial nerve-ligated group is 7 because one mouse died on day 2 after surgery due to an unknown cause.

Table 2. Comparison of pain-related score between three different experimenters

\begin{tabular}{|c|c|c|c|c|c|}
\hline \multirow[b]{3}{*}{ Experimenter } & \multicolumn{4}{|c|}{ Pain-related score (mean \pm S.E.M.) } & \multirow[b]{3}{*}{$\mathrm{n}$} \\
\hline & \multicolumn{2}{|c|}{ 1.6-mN von Frey filament } & \multicolumn{2}{|c|}{ 9.8-mN von Frey filament } & \\
\hline & Ligated & Contralateral & Ligated & Contralateral & \\
\hline A & $0.69 \pm 0.06^{*}$ & $0.08 \pm 0.04$ & $1.46 \pm 0.06^{*}$ & $0.77 \pm 0.06$ & 8 \\
\hline $\mathrm{B}$ & $0.50 \pm 0.04^{*}$ & $0.00 \pm 0.00$ & $1.53 \pm 0.11^{*}$ & $0.53 \pm 0.03$ & 6 \\
\hline $\mathrm{C}$ & $0.32 \pm 0.04^{*}$ & $0.02 \pm 0.01$ & $1.10 \pm 0.07^{*}$ & $0.27 \pm 0.04$ & 25 \\
\hline
\end{tabular}

The unilateral sural nerve was tightly ligated, and responses of both hind paws to stimulation with von Frey filaments were assessed on day 7 after ligation. $* P<0.05$ vs. contralateral paw (Wilcoxon signed rank test). 


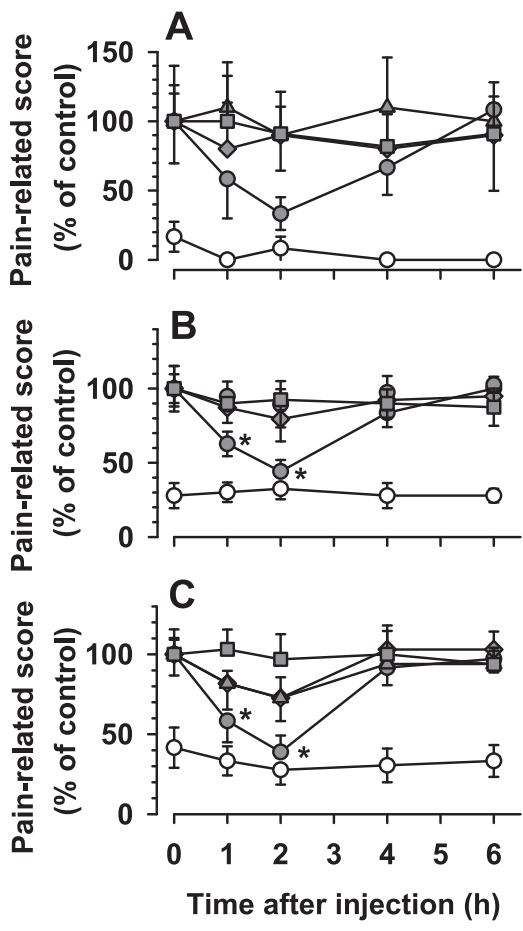

Fig. 3. Effects of gabapentin on pain-related behavioral responses to mechanical and cool stimuli in the mice that underwent sural nerve ligation. Gabapentin at doses of 3 (triangles), 10 (diamonds), and $30 \mathrm{mg} / \mathrm{kg}$ (circles) and vehicle (squares) were injected intraperitoneally 7 days after the sural nerve ligation. The hind paws were stimulated by von Frey filaments with bending force of 1.6 (A) or $9.8 \mathrm{mN}$ (B) or by acetone application (C). Closed symbols, ligated side; open symbols, contralateral side. Pain-related score was normalized to the score of the affected paw before gabapentin injection. The curves for the contralateral hind paws of the groups given 3 and $10 \mathrm{mg} / \mathrm{kg}$ gabapentin and vehicle were similar to that of $30 \mathrm{mg} / \mathrm{kg}$ of gabapentin and they are shown on the graphs. Each value is the mean and S.E.M. of $7-10$ animals. ${ }^{*} P<0.05$ vs. preinjection (Dunnett's multiple comparisons).

typical traces of firing in the WDR neuron evoked by mechanical (brush, 1.6 and $9.8 \mathrm{mN}$ filaments and pinch) and cool stimulation are shown in Fig. 4A. The sural nerve ligation significantly increased the responses to all kinds of stimuli examined (Fig. 4B).

\section{Effects of gabapentin on the activity of dorsal horn neurons}

Figure 5A shows typical examples of the effects of gabapentin $(30 \mathrm{mg} / \mathrm{kg})$ on the neuronal activity in mice given the sural nerve ligation. Although saline was without effects, gabapentin decreased the spontaneous activity and responses of the WDR neurons to mechanical and cool stimuli down to the level of the corresponding activity in naive mice; decreases in averaged firing were more marked at $2 \mathrm{~h}$ than $1 \mathrm{~h}$ after administration, and the levels of firing after $2 \mathrm{~h}$ were similar to those of naive mice (Fig. 5B). Gabapentin $(30 \mathrm{mg} / \mathrm{kg})$ was without

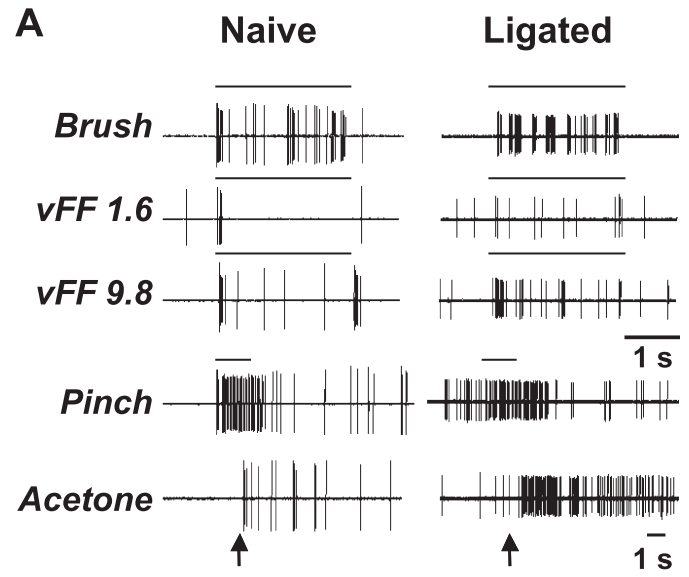

B

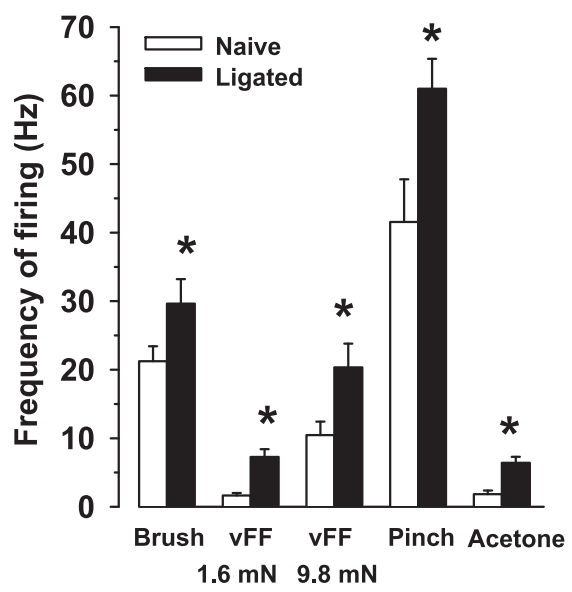

Fig. 4. Effects of sural nerve ligation on the responses of wide dynamic range neurons to mechanical and cool stimuli. The hind paw was stimulated by a brush, von Frey filaments (vFFs) with bending force of 1.6 or $9.8 \mathrm{mN}$, pinch, and acetone application. A) Typical examples of responses to the peripheral stimuli. Mechanical stimuli were given during the period shown by the lines over the charts, and cool stimuli were given at time shown by arrows. B) Quantitative comparison of responses to the peripheral stimuli. Values represent the mean and S.E.M. of 8 naive and 12 ligated animals. ${ }^{*} P<0.05$ vs. naive group (Student's $t$-test).

effects on the evoked responses of the WDR neurons in naive mice (Fig. 5B).

\section{Discussion}

The tight ligation of one of three branches (sural, tibial, and common peroneal nerves) of the sciatic nerve resulted in hypersensitivity to mechanical stimulation, although the intensity and time-course are not similar to each other. Ligation of the sural nerve markedly produced mechanical hypersensitivity without apparent effects on motor function of the hind paw. Sural nerve ligation in rats also does not affect motor function (18). Ligation of the tibial nerve also markedly produced 
A

\section{Pre (0 h) Post (2 h)}
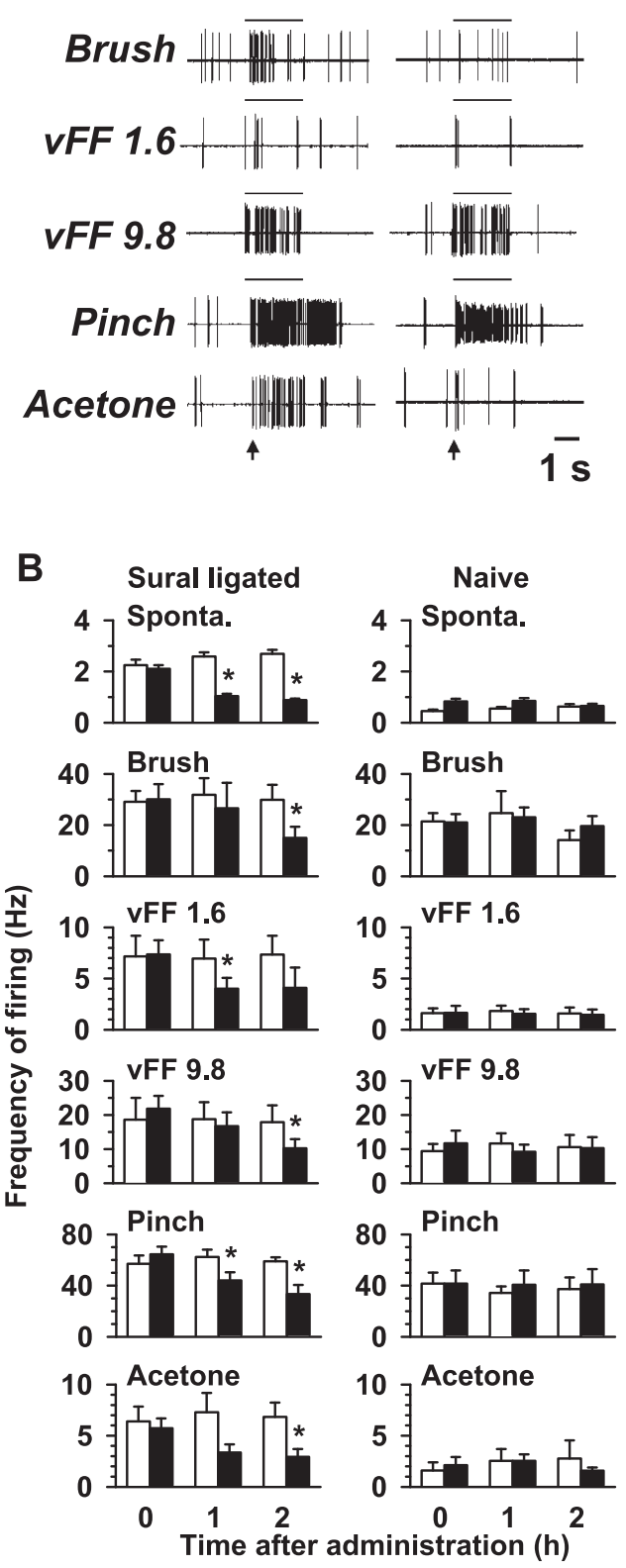

Fig. 5. Effects of gabapentin on the spontaneous activity and responses of wide-dynamic range neurons to mechanical and cool stimuli in naive and sural nerve-ligated mice. The hind paw was stimulated by a brush, von Frey filaments (vFFs) with bending force of 1.6 or $9.8 \mathrm{mN}$, pinch, and acetone application. Gabapentin at a dose of $30 \mathrm{mg} / \mathrm{kg}$ (closed columns; $\mathrm{n}=5$ and 7 for naive and ligated animals, respectively) and vehicle (open columns, $n=6$ for naïve and ligated animals) were injected intraperitoneally 7 days after sural nerve ligation. A) Typical example of gabapentin effects on the responses to the peripheral stimuli in the ligated mice. B) Quantitative comparison of spontaneous activity and responses to the stimuli. Each value is a mean and S.E.M. ${ }^{*} P<0.05$ vs. $0 \mathrm{~h}$ (Dunnett's multiple comparisons). mechanical hypersensitivity, but it affected the motor function of the hind paw. Ligation of the common peroneal nerve affected the motor function and mechanical hypersensitivity was the mildest. Seven to ten percent of the tibial and peroneal nerves are motor axons, but the sural nerve contains few motor axons (19). This may be a reason for the lack of effects of the sural nerve ligation on motor functions. The sural nerve may be most suitable for a mouse model of neuropathy of a sciatic nerve branch.

Injuries of the sural and tibial nerves produced mechanical allodynia in the tibial and common peroneal territories. Gabapentin $(30 \mathrm{mg} / \mathrm{kg})$ suppressed mechanical allodynia after sural nerve injury (present study), but it did not inhibit that of tibial nerve injury even at a dose of $100 \mathrm{mg} / \mathrm{kg}$ (20). Therefore, the pathologic conditions may be different between sural nerve and tibial nerve injuries. Ligation of the sural nerve produced cold and mechanical allodynia, which were almost completely inhibited by gabapentin $(30 \mathrm{mg} / \mathrm{kg})$. It is similar to spinal nerve ligation; it causes cold and mechanical allodynia, which are inhibited by gabapentin, although almost complete inhibition is observed after the dose of $300 \mathrm{mg} / \mathrm{kg}$ (13). The ligation of the tibial and common peroneal nerves also produces cold and mechanical allodynia, but gabapentin $(100 \mathrm{mg} / \mathrm{kg})$ significantly suppresses mechanical allodynia without inhibition of cold allodynia (14). Therefore, the pathologic conditions after sural nerve ligation are not the same as those after ligation of the tibial and common peroneal nerve.

Gabapentin $(30 \mathrm{mg} / \mathrm{kg})$ decreased the spontaneous activity and responses of WDR neurons to peripheral stimuli in mice that underwent sural nerve ligation down to the level of the corresponding activity in naive mice. The spontaneous activity and responses of WDR neurons were not inhibited by gabapentin at the same dose in naïve mice. Although the results are similar to those in a rat model of spinal nerve ligation injury, in which gabapentin $(20 \mathrm{mg} / \mathrm{kg})$ produced partial inhibition of the increased spontaneous activity and responses of WDR neurons without effects in naive animals (21), gabapentin is more effective in the sural nerve ligation model than in the spinal nerve ligation model.

Responses of the WDR neurons to all kinds of stimuli (tactile, noxious mechanical, and cool stimuli) were almost completely inhibited by gabapentin at a dose of $30 \mathrm{mg} / \mathrm{kg}$. Gabapentin acts on the $\alpha_{2} \delta$ subunit of voltage-dependent calcium channels $(22,23)$, which may be associated with reduction in transmitter release and neuronal activity. Gabapentin reduces the release of excitatory amino acids in the spinal cord following the inflammatory stimulation of the periphery $(24,25)$. It 
inhibits ectopic discharges of the sciatic nerve in a partial sciatic nerve ligation model (26). These findings taken together suggest that gabapentin acts on the primary afferents to suppress pain. The spinal nerve ligation induces the up-regulation of the $\alpha_{2} \delta$ subunit in the dorsal root ganglion, which is associated with the anti-allodynic effect of gabapentin (27). It is interesting to examine whether sural nerve ligation affects the expression of the $\alpha_{2} \delta$ subunit because gabapentin inhibited the hyperactivity of the WDR neurons in the operated, but not naive, animals. Recently, gabapentin has been shown to cause disinhibition of the locus coeruleus neurons, which results in the suppression of the activity of spinal dorsal horn neurons through the activation of the descending noradrenergic inhibitory system in rats with partial sciatic nerve ligation $(28,29)$. Such inhibitory mechanisms of gabapentin may also be involved in the analgesic effects and the inhibition of the hyperactivity of the WDR neurons in mice with sural nerve ligation.

In summary, the ligation of the sural nerve produced mechanical and cold allodynia without effect on motor function. It also increased the spontaneous activity and the responses of the WDR neuron to mechanical and cool stimulation. Gabapentin produced almost complete inhibition of behavioral allodynia and the hyperactivity of the WDR neurons in this model. Sural nerve ligation provides a new mouse model of neuropathic pain.

\section{Acknowledgment}

This work was supported partly by a Grant-in Aid for Exploratory Research (16650067) from the Ministry of Education, Culture, Sports, Science, and Technology, Japan.

\section{References}

1 Bennett GJ, Xie Y-K. A peripheral mononeuropathy in rat that produces disorders of pain sensation like those seen in man. Pain. 1988;33:87-107.

2 Seltzer Z, Dubner R, Shir Y. A novel behavioral model of neuropathic pain disorders produced in rats by partial sciatic nerve injury. Pain. 1990;43:205-218.

3 Kim SH, Chung JM. An experimental model for peripheral neuropathy produced by segmental spinal nerve ligation in the rat. Pain. 1992;50:355-363.

4 Kim KJ, Yoon YW, Chung JM. Comparison of three rodent neuropathic pain models. Exp Brain Res. 1997;113:200-206.

5 Wang LX, Wang ZJ. Animal and cellular models of chronic pain. Adv Drug Deliv Rev. 2003;55:949-965.

6 Holland NR, Crawford TO, Hauer P, Cornblath DR, Griffin JW, McArthur JC. Small-fiber sensory neuropathies: clinical course and neuropathology of idiopathic cases. Ann Neurol. 1998;44: $47-59$.

7 Zhou L, Kitch DW, Evans SR, Hauer P, Raman S, Ebenezer GJ, et al. Correlates of epidermal nerve fiber densities in HIVassociated distal sensory polyneuropathy. Neurology. 2007;68: 2113-2119.

8 Decosterd I, Woolf CJ. Spared nerve injury: an animal model of persistent peripheral neuropathic pain. Pain. 2000;87:149-158.

9 Bourquin AF, Suveges M, Pertin M, Gilliard N, Sardy S, Davison AC, et al. Assessment and analysis of mechanical allodynia-like behavior induced by spared nerve injury (SNI) in the mouse. Pain. 2006;122:14.e1-14.e14.

10 Backonja M, Beydoun A, Edwards KR, Schwartz SL, Fonseca $\mathrm{V}$, Hes $\mathrm{M}$, et al. Gabapentin for the symptomatic treatment of painful neuropathy in patients with diabetes mellitus: a randomized controlled trial. JAMA. 1998;280:1831-1836.

11 Rowbotham M, Harden N, Stacey B, Bernstein P, MagnusMiller L. Gabapentin for the treatment of postherpetic neuralgia: a randomized controlled trial. JAMA. 1998;280:1837-1842.

12 Attal N, Brasseur L, Parker F, Chauvin M, Bouhassira D. Effects of gabapentin on the different components of peripheral and central neuropathic pain syndromes: a pilot study. Eur Neurol. 1998;40:191-200.

13 Back SK, Won SY, Hong SK, Na KS. Gabapentin relieves mechanical, warm and cold allodynia in a rat model of peripheral neuropathy. Neurosci Lett. 2004;368:341-344.

14 Erichsen HK, Blackburn-Munro G. Pharmacological characterisation of the spared nerve injury model of neuropathic pain. Pain. 2002;98:151-161.

15 Hofmann HA, De VJ, Siegling A, Spreyer P, Denzer D. Pharmacological sensitivity and gene expression analysis of the tibial nerve injury model of neuropathic pain. Eur J Pharmacol. 2003;470:17-25.

16 Zimmermann M. Ethical guidelines for investigations of experimental pain in conscious animals. Pain. 1983;16:109-110.

17 Sasaki A, Takasaki I, Andoh T, Nojima H, Shiraki K, Kuraishi Y. Roles of $\alpha$-adrenoceptors and sympathetic nerve in acute herpetic pain induced by herpes simplex virus inoculation in mice. J Pharmacol Sci. 2003;92:329-336.

18 Van Remoortere MP, Meert TF, Vissers KC, Coppenolle H, Adriaensen H. Refinement of symptoms of neuropathic pain measurements after various transections of the nerve endings of the sciatic and femoral nerve in rats: an exploratory behavioral analysis. Anesth Analg. 2007;104:1236-1245.

19 Schmalbruch H. Fiber composition of the rat sciatic nerve. Anat Rec. 1986;215:71-81.

20 Hofmann HA, De Vry J, Siegling A, Spreyer P, Denzer D. Pharmacological sensitivity and gene expression analysis of the tibial nerve injury model of neuropathic pain. Eur J Pharmacol. 2003;470:17-25.

21 Matthews EA, Dickenson AH. A combination of gabapentin and morphine mediates enhanced inhibitory effects on dorsal horn neuronal responses in a rat model of neuropathy. Anesthesiology. 2002;96:633-640.

22 Gee NS, Brown JP, Dissanayake VU, Offord J, Thurlow R, Woodruff GN. The novel anticonvulsant drug, gabapentin (Neurontin), binds to the $\alpha_{2} \delta$ subunit of a calcium channel. J Biol Chem. 1996;271:5768-5776.

23 Field MJ, Cox PJ, Stott E, Melrose H, Offord J, Su TZ, et al. Identification of the $\alpha_{2}-\delta$-1 subunit of voltage-dependent calcium channels as a molecular target for pain mediating the analgesic actions of pregabalin. Proc Natl Acad Sci US A. 2006;103: 17537-17542. 
24 Feng Y, Cui M, Willis WD. Gabapentin markedly reduces acetic acid-induced visceral nociception. Anesthesiology. 2003;98: 729-733.

25 Coderre TJ, Kumar N, Lefebvre CD, Yu JS. Evidence that gabapentin reduces neuropathic pain by inhibiting the spinal release of glutamate. J Neurochem. 2005;94:1131-1139.

26 Pan HL, Eisenach JC, Chen SR. Gabapentin suppresses ectopic nerve discharges and reverses allodynia in neuropathic rats. $\mathrm{J}$ Pharmacol Exp Ther. 1999;288:1026-1030.

27 Luo ZD, Chaplan SR, Higuera ES, Sorkin LS, Stauderman KA, Williams ME, et al. Upregulation of dorsal root ganglion $\alpha_{2} \delta$ calcium channel subunit and its correlation with allodynia in spinal nerve-injured rats. J Neurosci. 2001;21:1868-1875.

28 Tanabe M, Murakami H, Honda M, Ono H. Gabapentin depresses C-fiber-evoked field potentials in rat spinal dorsal horn only after induction of long-term potentiation. Exp Neurol. 2006;202:280-286.

29 Tanabe M, Takasu K, Kasuya N, Shimizu S, Honda M, Ono H. Role of descending noradrenergic system and spinal $\alpha_{2}-$ adrenergic receptors in the effects of gabapentin on thermal and mechanical nociception after partial nerve injury in the mouse. Br J Pharmacol. 2005;144:703-714. 